\title{
ANGIOGENIC COMPETENCY OF BIODEGRADABLE HYDROGELS FABRICATED FROM POLYETHYLENE GLYCOL-CROSSLINKED TYROSINE-DERIVED POLYCARBONATES
}

\author{
H.J. Sung, K.M. Sakala Labazzo, D. Bolikal, M.J. Weiner, R. Zimnisky, J. Kohn*
}

New Jersey Center for Biomaterials, Rutgers, The State University of New Jersey, 145 Bevier Road, Piscataway, NJ 08854, USA

\begin{abstract}
Synthetic biomaterials can be used as instructive biological milieus to guide cellular behaviour and function. To further realize this application, we synthesized a series of structurally similar hydrogels and tested their ability to modulate angiogenesis. Hydrogels were synthesized from poly(DTE-co- $x \%$ DT carbonate) crosslinked by $y \%$ poly(ethylene glycol) (PEG). Hydrogel desaminotyrosyl tyrosine (DT) contents $(x \%)$ ranged from $10-100 \%$, and crosslink densities ( $y \%$ PEG-crosslinker) ranged from 5$80 \%$. The hydrogels were fashioned into porous scaffolds with highly interconnected macro- and micro-pore ( $>100$ and $<10 \mu \mathrm{m}$ in diameter, respectively) architecture using poly(DTE-co-10\%DT carbonate) crosslinked with $8 \%$ PEG. Under physiological conditions (in vitro), the hydrogels degraded into three major products: desaminotyrosyltyrosine ethyl ester (DTE), desaminotyrosyl tyrosine (DT), and poly(ethylene glycol)-di-DT-hydrazide (PEG-di-DT hydrazide). Increasing either DT content or crosslink density brought quickened degradation. Because DT and DTE, two of the three major degradation products, have not demonstrated any noticeable cytotoxicity or angiogenic effect in previous studies, we measured the cytotoxicity of PEG-di-DT hydrazide, the third major degradation product. We found that PEG-di-DT hydrazide only displayed significant cytotoxicity at the high concentration of 100 $\mathrm{mg} / \mathrm{mL}$. Interestingly, PEG-di-DT hydrazide and its further degradation product PEG-dihydrazide stimulated in vitro endothelial cell migration and tubulogenesis, which is comparable to results found with FGF- $\beta$ treatment. Subcutaneous implantation of the PEG-crosslinked poly(DTE-co- $10 \%$ DT carbonate) scaffolds into the backs of rats elicited greater tissue growth over time and superior vascularization than poly(DTE carbonate) implantation. These results show that this new class of biomaterials has a strong potential to modulate angiogenesis.
\end{abstract}

Keywords: Tyrosine-derived polycarbonates, PEGcrosslink, hydrogel, degradation, angiogenesis.

*Address for correspondence:

Joachim Kohn

New Jersey Center for Biomaterials, Rutgers,

The State University of New Jersey,

145 Bevier Road, Piscataway, NJ 08854, USA

Telephone Number: 001-732-445-0488

FAX Number: 001-732-445-5006

E-mail: kohn@biology.rutgers.edu

\section{Introduction}

Tyrosine-derived polycarbonates are a group of homologous carbonate-amide copolymers individually differing only in the length of their respective alkyl ester pendant chains (Fig. 1) (Ertel and Kohn, 1994). Various polymers of desaminotyrosyl-tyrosine alkyl esters (DTR) have been extensively studied and characterized as tissue compatible, strong, hydrophobic, and slow degrading materials (Ertel and Kohn, 1994; James et al., 1999; Yu and Kohn, 1999). Out of the DTR polymers studied, Poly desaminotyrosyl-tyrosine ethyl ester (DTE) carbonate has shown the most promise for biocompatibility. Recently, the incorporation of free carboxylate pendent chains, desaminotyrosyl tyrosine (DT), into poly(DTR carbonate)s has been shown to increase the degradation rate (Abramson, 2002).

A series of copolymers with the general formula poly(DTE-co- $x \%$ DT carbonate) were created with DT contents ranging 5-100 mole $\%$. This polymer series possesses higher degradation rates than the poly(DTR carbonate)s, while still maintaining the mechanical properties and processability of poly(DTR carbonate)s. Through semicarbazide linkage, poly(DTE-co- $x \% \mathrm{DT}$ carbonate)s can be chemically crosslinked with poly(ethylene glycol)-dihydrazide (PEG-dihydrazide) (Fig. 1b) at the free acid groups of the DT monomer. The incorporation of PEG increases the hydrolytically degradable property (Zhao and Harris, 1998) of the polymer by increasing the hydrophilicity. Altering the level of PEG crosslinking significantly changes the material properties. By increasing the amount of crosslinking, the polymer scaffolds can change from a stiff, hydrophobic material into a soft, hydrophilic hydrogel.

Angiogenesis is the growth of new blood vessels from the existing vascular system (Bishop et al., 1999; Folkman and Shing, 1992; Lutolf and Hubbell, 2005). This process occurs naturally during reproduction, wound repair, placental development, and the foreign body response. New methods of modulating and directing angiogenesis, including the utilization of endothelial growth factors or the use of polymeric biomaterials, provide new treatment options for the fields of regenerative medicine and wound healing (Black et al., 1999; Cassell et al., 2002; TelloMontoliu et al., 2006; Yla-Herttuala et al., 2007). For biomaterial tissue scaffolds, it is crucial that the scaffolds permit proper vascularization so as to provide functional tissue with adequate nutrients and oxygen (Jain et al., 1997; Sieminski and Gooch, 2000). Endothelial cells (ECs) grown on a biopolymeric matrix curl, associate, 
a)<smiles>CC(C)(C)Oc1ccc(CCC(=O)NC(Cc2ccc(OC(C)(C)C)cc2)C(=O)O)cc1</smiles>

b)<smiles>CC(C)(CC(C)(C)C(=O)NN)OC(=O)NN</smiles>

c)

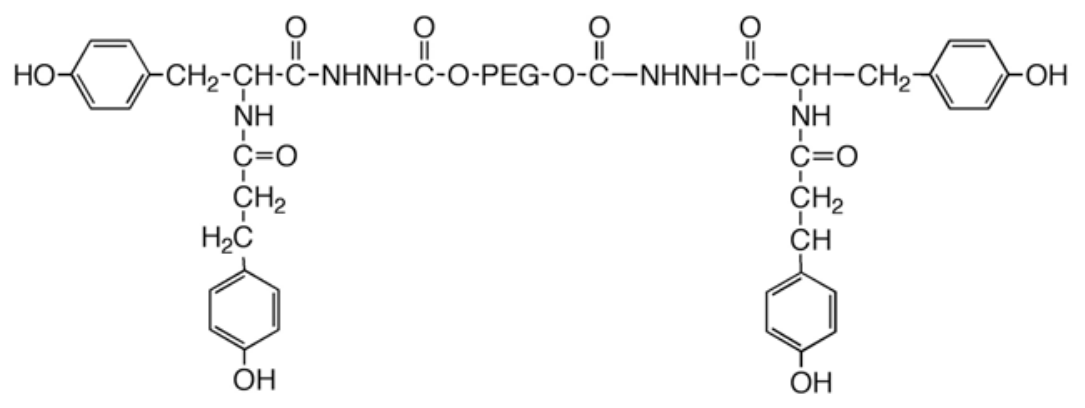

Figure 1. Polymer structure. a) Tyrosine-derived polycarbonates: the monomer is desaminotyrosyl-tyrosine alkyl ester (DTR). Alkyl ester (R) pendent chain can be designed to ethyl (DTE), butyl (DTB), hexyl (DTH) and octyl (DTO). b) PEG-dihydrazide. c) Model compound, PEG-di-DT hydrazide.

and elongate into tubule structures that resemble immature budding capillaries during angiogenesis (Bachetti and Morbidelli, 2000). EC proliferation and migration are crucial events in angiogenesis (Bernardini et al., 2005).

Several strategies have been employed to induce vascularization with biomaterial implants, including the incorporation of biological moieties like growth factors, the functionalization of materials to absorb angiogenic factors to the implant site, and the use of cell lines that secrete angiogenic substances (Eckardt et al., 2003; Kidd et al., 2002; Tabata et al., 1998). However, there are some problems with current strategies. For instance, undesired tissue growth and increased vessel permeability are potential side effects; there is also a general lack of information regarding other biological activities relating to these strategies. Therefore, there is great need for a new synthetic polymer with controlled and predictable angiogenic activity based on the material properties.

One reason why clinical researchers go to such lengths to increase new blood vessel growth around an implant is that capillaries act as highways to transport cell metabolites to and from the site and aid in inflammatory cell action. The disruptive action of surgically implanting a material into the body creates trauma. If an implant site is better suited for dealing with inflammatory cells and the body's natural healing response because of increased blood flow due to angiogenesis, there is a better chance that implant patency will remain preserved. In other words, a tissue engineered implant should be able to perform its function effectively (i.e. vascular grafts remain open, bone grafts support mechanical loading, and native tissue infiltration should occur on a timescale to match the degradation of degradable polymer scaffolds) for the lifetime of the implant. For tissue engineered scaffolds used as dermal equivalents, some researchers realized that seeding endothelial cells directly into the scaffold with keratinocytes led to better vascularization of the natural polymer scaffolds and resulted in greater cell growth.

To further expand the properties and the utility of the tyrosine-derived polycarbonates, we fabricated a series of biodegradable hydrogels from PEG-crosslinked poly(DTE-co-DT carbonate)s. The degradation profile of the resulting materials exemplified unique, controlled, predictable kinetics. The hydrogels and their degradation products exhibited suitable biocompatibility and great potential to modulate angiogenesis.

\section{Methods}

\section{Polymer synthesis, scaffold fabrication, and characterization}

\section{Materials}

$\mathrm{N}, \mathrm{N}$-dimethylformamide (DMF), poly(ethylene glycol) (PEG), Amberlyst 15, Trizma buffer solution, and Diff Quik staining were obtained from Sigma-Aldrich (St. Louis, MO, USA). N-hydroxysuccinimide (NHS), triethylamine (TEA), hydrazine, and hydroxybenzotriazole (HOBt) were obtained from Acros Organics (Morris Plains, NJ, USA). N-ethyl-N'-dimethylaminopropyl carbodiimide. $\mathrm{HCl}$ (EDCI) was obtained from Tanabe Research Laboratories U.S.A., Inc. (San Diego, CA). HPLC grade solvents were used for HPLC analysis and other work unless otherwise stated. DMF and 1-methylpyrrolidinone (Omnisolv, EM Science, Gibbstown, NJ, USA) were purified by treatment with Amberlyst 15 to remove basic impurities. 
Table 1. Stoichiometric amounts of PEG-dihydrazide and EDCI used to fabricate non-porous and porous scaffolds. (Unit: mg)

\begin{tabular}{|c|c|c|c|c|}
\hline \multirow{2}{*}{ Hydrogels } & \multicolumn{2}{|c|}{ Non-porous scaffolds } & \multicolumn{2}{c|}{ Porous scaffolds } \\
\cline { 2 - 5 } & PEG-diH & EDCI & PEG-diH & EDCI \\
\hline $10 \mathrm{D} / 8 \mathrm{P}$ & 446 & 80 & 67 & 12 \\
\hline $15 \mathrm{D} / 12 \mathrm{P}$ & 670 & 120 & 101 & 18 \\
\hline $20 \mathrm{D} / 16 \mathrm{P}$ & 900 & 162 & 135 & 24 \\
\hline $25 \mathrm{D} / 5 \mathrm{P}$ & 282 & 51 & 84 & 15 \\
\hline $25 \mathrm{D} / 20 \mathrm{P}$ & 1130 & 203 & 168 & 30 \\
\hline $50 \mathrm{D} / 20 \mathrm{P}$ & 1140 & 207 & 172 & 31 \\
\hline $100 \mathrm{D} / 20 \mathrm{P}$ & 1200 & 215 & 179 & 32 \\
\hline $100 \mathrm{D} / 80 \mathrm{P}$ & 4770 & 862 & 716 & 129 \\
\hline
\end{tabular}

\section{Preparation of the crosslinker, PEG-dihydrazide}

One hundred grams of PEG (Mw=2000 g/mol, $0.05 \mathrm{~mol})$ was dissolved in $1 \mathrm{~L}$ of toluene and then dried azeotropically; $150 \mathrm{~mL}$ of $20 \%$ phosgene in toluene was then added. The mixture was then stirred overnight to produce PEG-dichloroformate (excess phosgene and toluene were distilled off). One hundred $\mathrm{mL}$ of methylene chloride and $300 \mathrm{~mL}$ of dry toluene were then added to the residue. Seventeen grams of N-hydroxysuccinimide (NHS) $(0.15 \mathrm{~mol})$ and 15 grams of triethylamine (TEA) $(0.15 \mathrm{~mol})$ were subsequently added to the residue; stirring resumed for 2 hours at $0^{\circ} \mathrm{C}$. Next, $15.7 \mathrm{~mL}$ of Hydrazine $(0.50 \mathrm{~mol})$ was added to the reaction mixture, which was stirred for an additional 12 hours. The precipitate of $\mathrm{TEA} \times \mathrm{HCl}$ was removed by filtration; the filtrate was concentrated to a volume of $200 \mathrm{~mL}$; and PEG-dihydrazide was precipitated with $600 \mathrm{~mL}$ of diethyl ether. Then, the desired product, PEG-dihydrazide, was isolated by filtration, recrystallized using $800 \mathrm{~mL}$ of isopropanol (IPA), and finally dried under vacuum at room temperature. ${ }^{1} \mathrm{H}$ NMR was used to determine the structure and purity of the product.

\section{Crosslinking and scaffold fabrication}

Poly(DTE-co-X\%DT carbonate)s were synthesized as previously described (Abramson, 2002). For the scaffold, $0.3 \mathrm{~g}$ of the polymer was dissolved in $3 \mathrm{~mL}$ of $\mathrm{N}$ Methylpyrrolidone (NMP), and the corresponding amounts of PEG-dihydrazide and EDCI/methylene chloride were added (Table 1). After two minutes of mixing in a vortex mixer, the solution was poured into a Teflon dish $5 \mathrm{~cm}$ in diameter. To prepare porous scaffolds, $10 \mathrm{~g}$ of sieved $\mathrm{NaCl}$ crystals $(212-425 \mu \mathrm{m})$ (Sung et al., 2004) were placed into the Teflon dish; then, the polymer solution was carefully poured over the salt bed. To produce micropores less than $10 \mu \mathrm{m}$ in diameter, the sample dish was immersed in liquid nitrogen for 30 minutes to induce phase separation (Levene, 1999). The scaffolds were repeatedly washed with deionized water to remove $\mathrm{NMP}, \mathrm{NaCl}$, and other byproducts. By varying polymer DT content and PEG- dihydrazide stoichiometry, a series of hydrogels with differing degrees of crosslinking (from 5-80\%) and DT percents (from 10-100\%) were prepared. The hydrogel composition is presented as $x \mathrm{D} / y \mathrm{P}$ where $x$ represents the $\% \mathrm{DT}$ and $y$ represents the degree of crosslinking (\%PEGdihydrazide). For example, 10D/8P represents poly(DTEco- $10 \%$ DT carbonate) with $8 \%$ crosslinking. This nomenclature will be used throughout the manuscript.

Synthesis of the model degradation compound PEG-

\section{di-DT Hydrazide}

Over an ice-water bath, $4.5 \mathrm{mmol}$ of DT, $2.36 \mathrm{mmol}$ of PEG-dihydrazide, $0.45 \mathrm{mmol}$ of $\mathrm{HOBt}$, and $10 \mathrm{~mL}$ of NMP in methylene chloride were mixed and reacted for 1 hour. Next, $4.72 \mathrm{mmol}$ of EDCI was reacted to the mixture by stirring for 24 hours. The reaction mixture was then washed with a solution of $5 \%$ sodium bicarbonate, $0.2 \mathrm{M}$ hydrochloric acid, and $20 \%$ sodium chloride in water. The organic phase was dried over magnesium sulphate, filtered, and then evaporated. We dissolved the resulting oil in IPA $(50 \mathrm{~mL})$ under heat and then crystallized the product at

$-20^{\circ} \mathrm{C}$. The PEG-di-DT hydrazide yellow solid was washed with IPA and dried under vacuum at $40^{\circ} \mathrm{C}$.

\section{Scaffold imaging}

Hydrogels were prepared for Scanning Electron Microscope (SEM) analysis by immersion in liquid nitrogen until frozen and were then freeze-fractured. Prior to SEM analysis, samples were dried under vacuum for 24 hours, mounted on aluminium studs with adhesive paper, and sputter-coated with gold/palladium under vacuum for 120 seconds. Samples were visualized on an AMRAY 1830-I Scanning Electron Microscope at $20 \mathrm{kV}$. For optical coherence tomography (OCT), samples were placed in a test tube and evacuated for $10 \mathrm{~min}$ to remove air. Distilled water was introduced while the samples were still under vacuum. The scaffolds were placed on a Petri dish and immersed in distilled water for imaging. Samples were imaged on an OCT using the $26 \mathrm{X}$ objective having a resolution of $21 \mu \mathrm{m}$ in the $\mathrm{x}$ direction. 


\section{Degradation}

\section{Water uptake}

Dried, non-porous hydrogels $8 \mathrm{~mm}$ in diameter and $2 \mathrm{~mm}$ thick were weighed (weight $\left.{ }_{d r y}\right)$, incubated in water at room temperature for 48 hours, and re-weighed to obtain the wet weight $\left(\right.$ weight $\left._{\text {wet }}\right)$. The percent water uptake $(\mathrm{n}=5)$ was calculated as:

$$
\% \text { wateruptake }=\frac{\text { weight }_{\text {wet }}-\text { weight }_{d r y}}{\text { weight }_{d r y}} \times 100
$$

\section{Mass loss}

Non-porous hydrogel scaffolds were weighed (weight ${ }_{\text {init }}$ ) and then incubated at $37^{\circ} \mathrm{C}$ with a Trizma buffer solution ( $\mathrm{pH} 7.4$, Sigma) that was modified by the addition of $\mathrm{HCl}$, $\mathrm{NaCl}, \mathrm{KCl}, \mathrm{NaHCO}_{3}, \mathrm{MgCl}_{2} \bullet 6 \mathrm{H}_{2} \mathrm{O}, \mathrm{MgSO}_{4}, \mathrm{~K}_{2} \mathrm{HPO}_{4}$, and $\mathrm{CaCl}_{2}$ to simulate the composition of body fluid (Labazzo, 2004). Hydrogels were vacuum-dried for two weeks and re-weighed (weight $t_{t p}$ ) at nine different time-points: 3 days, $1,2,3,4,6,12,18$, and 24 weeks. Percent mass loss $(n=5)$ was calculated as:

$$
\% \text { massloss }=\frac{\text { weight }_{\text {init }}-\text { weight }_{t p}}{\text { weight }_{\text {init }}} \times 100
$$

\section{Analysis of degradation product}

By using HPLC (Perkin-Elmer, Norwalk, CA, USA), buffers collected from the mass loss measurements at like time points were analyzed, and the cumulative release (mg) of DT from non-porous scaffolds was measured. Further degradation of PEG-di-DT hydrazide was analyzed after incubation at $37^{\circ} \mathrm{C}$ with the modified Trizma buffer solution as described above. Desaminotyrosine (DAT) was used as an internal standard.

\section{In vitro and in vivo biological evaluation}

For biological evaluation, all materials were UV-sterilized for 30 minutes before use; solutions, which could not be sterilized, were filtered through a $0.22 \mu \mathrm{m}$ bacterial filter (Becton Dickinson Labware Europe, Le Pont de Cles, France). The hydrogels were prepared under aseptical conditions and UV-sterilized for 30 minutes before implantation.

\section{In vitro cytotoxicity, migration, and tubulogenesis}

Rat lung fibroblasts (RLF-6) were treated for 24 hours with varying concentrations of PEG-di-DT hydrazide, PEG, or lactic acid. Cytotoxicity was determined by using an MTS assay (Promega, Madison, WI) and following the instructions provided by the supplier (Malich et al., 1997). Human aortic endothelial cells (HAEC) purchased from Cambrex (Walkersville, MD, USA) were cultured in EGM2 medium according to the supplier's instructions. HAEC were derived from 3 individual donors. Cell cultures (P4P6) were maintained at $37^{\circ} \mathrm{C}, 5 \% \mathrm{CO}_{2}$ and $100 \%$ humidity. Migration assays were performed using a Costar microchemotaxis transwell chamber. The wells were coated with $10 \mu \mathrm{g} / \mathrm{mL}$ fibronectin (Calbiochem, San Diego, CA, USA). HAEC were seeded onto the transwells with one of the target molecules and then incubated for 6 hours. The migrated cells were stained with Diff Quik nucleus staining (Sigma) and counted under a light microscope.
Basic fibroblast growth factor $\beta$ (FGF- $\beta, 0.1 \mu \mathrm{g} / \mathrm{ml}$ ) was treated as a positive control (Bachetti and Morbidelli, 2000; Black et al., 1999; Cassell et al., 2002; Tabata et al., 1998).

We performed an in vitro matrigel tubulogenesis assay. We first incubated matrigel for complete gelation in a 96well plate at $37^{\circ} \mathrm{C}$ for 30 minutes. HAECs $\left(2 \times 10^{4} /\right.$ well $)$ were then seeded to the matrigel and treated with a target molecule for 18 hours (Montanez et al., 2002). The cells were stained with Diff Quick staining (Sigma) and imaged under a light microscope with a $4 \mathrm{X}$ objective lens. All images were processed semi-automatically using Microsuite software (AnalySIS, Olympus, Münster, Germany). The AnalySIS software enabled us to draw lines following the tubulogenic feature on the image and automatically calculated the interactive length of tubules. The quantitative data were then exported as an Excel format. FGF- $\beta(1 \mu \mathrm{g} / \mathrm{ml})$ was used as a positive control.

All the in vitro assays were performed using 4 replicates of each substrate type $(n=4)$, the minimum sample number suggested by Power Analysis with which statistical significance was valid. We statistically analyzed samples by comparison to the control condition treated by only culture media using one-way ANOVA testing with Dunnet post hoc analysis (confidence interval $95 \%$ or $p \leq 0.05$ ).

In vivo histological analysis

Porous 10D/8P and poly(DTE carbonate) scaffolds were implanted subcutaneously into the backs of rats $(n=4)$ and harvested 1, 3, 8, and 15 weeks post implantation. We stained the samples using haematoxylin and eosin (H\&E) for histological analysis.

\section{Results}

\section{Synthesis, fabrication, and characterization}

We synthesized PEG-dihydrazide, the chemical crosslinker, and obtained a yield of $81 \%$. We verified the chemical structure of the crosslinker using $1 \mathrm{H}$ NMR. The values in ${ }^{1} \mathrm{H} \mathrm{NMR}\left(\mathrm{CDCl}_{3}\right) \delta$ ppm relative to TMS were 6.71 (broad multiplet, $2 \mathrm{H}, \mathrm{NHNH}_{2}$ ), 4.27 (m, 4H, $\mathrm{CH}_{2}-\mathrm{O}-$ $\mathrm{C}=\mathrm{O})$, and $3.64\left(\mathrm{~m}, 176 \mathrm{H}\right.$, internal $\mathrm{CH}_{2} \mathrm{~s}$ of $\mathrm{PEG}$ and $\mathrm{NHNH}_{2}$ ).

We prepared the model degradation product, PEG-diDT hydrazide (Fig. 1c), with a yield of 42\%; HPLC analysis identified the purity of this compound to be $100 \%$. The values in ${ }^{1} \mathrm{H}$ NMR (DMSO) $\delta$ ppm relative to TMS were 9.86 and 9.16 (singlet, 2H, OHs), 9.13-9.09 (s, 4H, NHNH-), 8.03 (doublet, 2H, NH-CH), 7.02-6.59 (m, 16H, aromatic $\mathrm{Hs}), 4.46(\mathrm{~m}, 2 \mathrm{H}, \mathrm{CH}), 4.09(\mathrm{~m}, 4 \mathrm{H}$, terminal $\mathrm{CH}_{2}$ of PEG), 3.3 ( $\mathrm{m}, 176$, internal $\mathrm{CH}_{2} \mathrm{~s}$ of PEG), 2.75 (m, 4H, CH-CH $)$, and 2.99-2.25 (m, 8H, $\left.\mathrm{CH}_{2}-\mathrm{CH}_{2}-\mathrm{C}=\mathrm{O}\right)$.

The pore architecture of the $10 \mathrm{D} / 8 \mathrm{P}$ hydrogel scaffold was observed with SEM and OCT (Fig. 2). The SEM images show the macro-pores, formed by $\mathrm{NaCl}$ porogens, to be greater than $100 \mu \mathrm{m}$ in diameter (Fig. 2a). The micropores less than $10 \mu \mathrm{m}$ in diameter were produced by phase separation (Fig. 2b). OCT imaging shows a highly overlapped porous area (red colour) within a primarily nonporous area (green colour) (Fig. 2c), indicating an interconnected pore structure. 


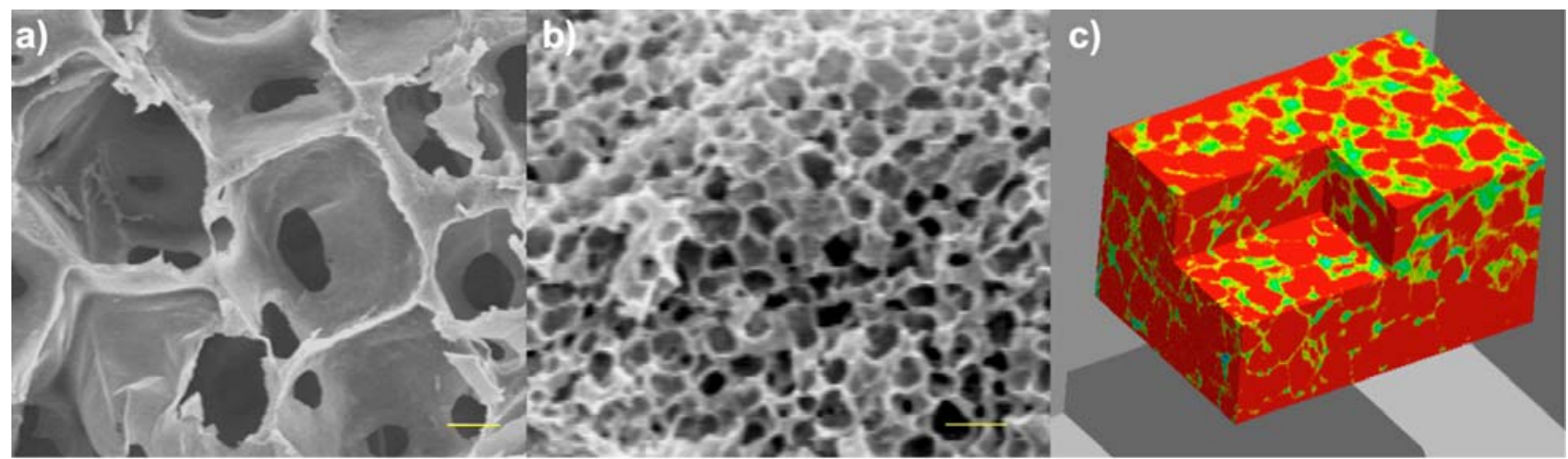

Figure 2. Pore architecture of the 10D/8P hydrogel scaffold. SEM images demonstrate a) macropores $($ Bar $=100$ $\mu \mathrm{m})$ and $\mathbf{b})$ micro-pores $(\mathrm{Bar}=10 \mu \mathrm{m})$. c) OCT image shows the interconnected pores. Red and green colour represents the porous and the non-porous area of the scaffold, respectively.

The design of these hydrogels provides for four distinct parameters that can be widely modified. The first parameter is the amount of free carboxylate pendent groups designated as DT. The DT content can vary from 0-100 mole $\%$; this indicates that none or all of the polymer repeat units carry a carboxylic acid group. The second parameter is the theoretical crosslink density (TCD). The TCD is determined by the amount of PEG-dihydrazide added to the reaction mixture during the crosslinking reaction. For example, when poly(DTE-co-50\%DT carbonate) was selected as the base polymer, $50 \mathrm{~mole} \%$ of all the polymer repeat units were DT, while 50 mole\% were unreactive DTE. For the crosslinking reaction, we used an amount of PEG-dihydrazide equivalent to $40 \mathrm{~mole} \%$ of the repeat units of poly(DTE-co-50\%DT carbonate) present in the reaction mixture. The resulting hydrogel had, theoretically, a PEG-dihydrazide crosslink at 40 mole $\%$ of all repeat units. A residual amount, $10 \mathrm{~mole} \%$, of the repeat units still carried a reactive carboxylate group (DT). The third design parameter is the molecular weight of the PEG chain used in the crosslinking reaction. The last parameter is the structure of the alkyl ester pendent chain used to block potentially reactive carboxylate groups. For this study, the DT content in the polymers ranged from $10-100 \%$, the TCD values ranged from 5-80\%, the PEG molecular weight was kept constant at $2000 \mathrm{~g} / \mathrm{mol}$, and the ethyl ester was used for all hydrogels studied.

\section{Degradation}

In order to exclude the influence of pores on degradation and focus solely on chemical composition, only non-porous films were tested in the degradation study. The degree of swelling ranged from $27-407 \%$. Out of the 8 hydrogels tested, the greatest amount of water uptake was observed with the 100D/80P and 100D/20P hydrogels (Fig. 3a). We used a 2-way ANOVA to determine the cause of the variation among the paired samples; DT content was found to be a significant contributor to the disparities in water uptake $(p<0.0001)$. In contrast, the crosslink density alone was not shown to significantly impact water uptake $(p>0.1)$. In fact, crosslinked polymers were only shown to deviate from the control's water uptake percentage when they contained DT, indicating that the primary determinant of water uptake was the DT content. This is especially obvious in the hydrogels that possessed equal degrees of crosslinking but had different DT contents (i.e. 100D/20P, 50D/20P, 25D/20P) (Fig 3a). Because DT has free acid groups that contribute to water uptake (Kim et al., 1999), decreasing the DT content is suspected to decrease polymer water uptake.

We measured the scaffold's percent mass loss over time in order to determine the degree of degradation into watersoluble fragments (Fig. 3b). The deviations and the large errors in Figure $3 \mathrm{~b}$ could be attributed to the rapid erosion of the degrading hydrogels, which makes accurate weighing of samples at each point in time difficult. The samples with high DT content (50\% and 100\% DT) lost most of their mass within 28 days. We analyzed the incubation solution by means of HPLC to determine the amount of DT released from the non-porous scaffolds. Figure $3 \mathrm{c}$ shows the cumulative DT release in $\mathrm{mg}$ calculated over time. Similar in nature to the mass loss results, the samples with high DT content $(50 \%$ or $100 \%$ DT) were found to release DTs rapidly for the first 21 days before exhibiting a deceleration in DT release. All of the other samples released DTs rapidly in a log phase manner within the first 40 days before entering a lag phase in DT release; a small amount of DT remained constantly present on the HPLC for the entire duration of the study. The 15D/ 12P hydrogel released fewer DTs than the other samples with higher DT contents, confirming DT's effect on degradation.

By using the HPLC to analyze the incubation solution, which housed the porous hydrogel scaffolds, we determined the retention times of the three major degradation products - DT, DTE, and PEG-di-DT hydrazide - from the different porous hydrogels. The HPLC retention time for a component in a sample is determined by measuring the time it takes for the peak representing the component to appear after the sample is injected. The retention time values, which are automatically calculated by the HPLC, will generally vary slightly. We found the DT retention times to range from 6.4 to 6.9 minutes for the different samples. The next product to be released, DTE held retention times of 8.7 and 8.9 minutes from the 50D/40P and 25D/20P hydrogels, respectively. No DTE was detected in the $100 \%$ DT hydrogel. PEG-diDT hydrazide, the final product to be released, held 
a)

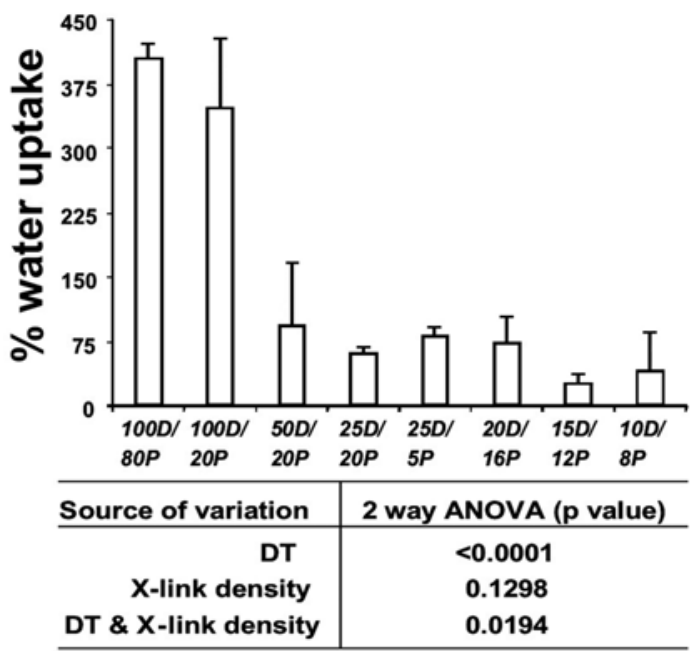

c)

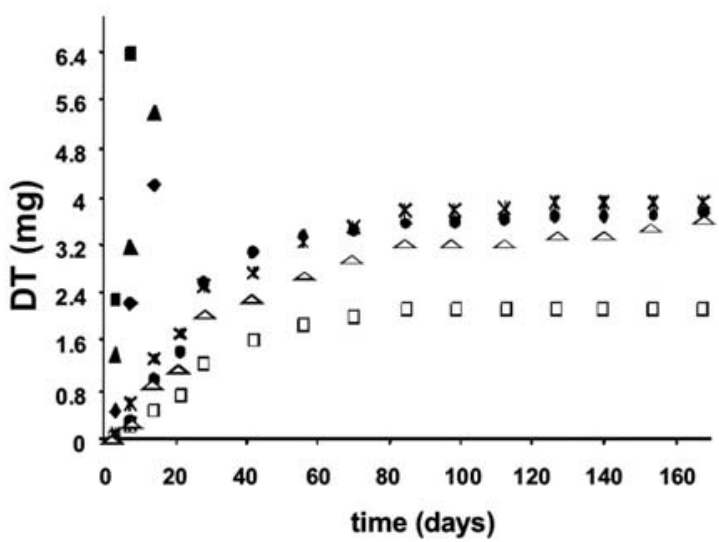

retention times ranging from 13.0 to 13.7 minutes. The further degradation of PEG-di-DT hydrazide was analyzed using the HPLC chromatogram, which showed no sign of any degradation products for approximately two weeks before it indicated degradation by a peak. Our analysis using the standard compounds determined this peak to be DT-NHNH ${ }_{2}$. The extended incubation of DT- $\mathrm{NHNH}_{2}$ at $37^{\circ} \mathrm{C}$ eventually revealed a DT peak on the HPLC. After about six and a half weeks, the DT- $\mathrm{NHNH}_{2}$ was nearly undetectable, and the DT peak continued to rise over the remainder of the study.

\section{In vitro and in vivo biological evaluation}

Because DT and DTE demonstrated no noticeable cytotoxicity or angiogenic effect in our previous studies (Ertel and Kohn, 1994; James et al., 1999; Yu and Kohn, 1999), the present study focused on the cytotoxic and angiogenic effect of PEG-di-DT hydrazide and its further degradation product, PEG-dihydrazide. Figure $4 \mathrm{a}$ illustrates the results of our measure of cytoxicity with an MTS assay. PEG-di-DT hydrazide and PEG were observed to significantly reduce RLF-6 viability when compared to the media $(p<0.05)$ but only when present at the highest concentration $(100 \mathrm{mg} / \mathrm{mL})$. However, at concentrations above $6.25 \mathrm{mg} / \mathrm{mL}$, lactic acid lowered the MTS value to the baseline to indicate significantly greater cytotoxicity than that of the media $(p<0.05)$.

We studied the effect of the degradation products on HAEC migration and compared their effect to that of the medium control (Figs. $4 \mathrm{~b}$ and $4 \mathrm{c}$ ). We began by testing b)

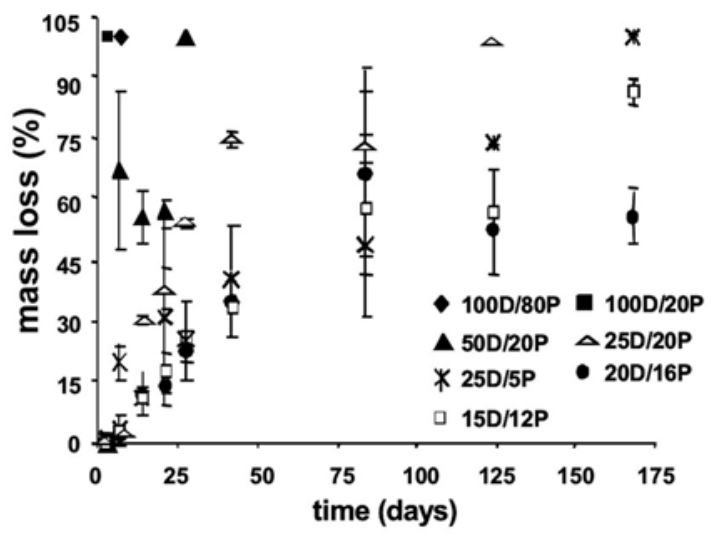

Figure 3. Degradation properties of non-porous hydrogel scaffolds. a) Percent water uptake of scaffolds for 48 hours. The source of the variation for percent water uptake was analyzed using two-way ANOVA. b) Mass loss and c) cumulative release of degraded DT from non-porous scaffolds measured over 168 days.

the dose response of HAEC migration to PEG-di-DT hydrazide treatment (Fig. 4b). FGF- $\beta$ was used as a positive control to induce migration. HAEC migration increased in response to the increase in PEG-di-DT hydrazide concentration from 0 to $50,000 \mu \mathrm{g} / \mathrm{mL}$. At concentrations of $1,000 \mu \mathrm{g} / \mathrm{mL}$ and above, ECs migrated significantly more than when they were incubated in the media free of PEG-di-DT hydrazide $(p<0.05)$. EC migration was comparable to the FGF- $\beta$ treatment at $0.1 \mu \mathrm{g} / \mathrm{mL}$; there was no statistical difference in EC migration between the PEG-di-DT hydrazide treated media and the media with $0.1 \mu \mathrm{g} / \mathrm{mL}$ FGF- $\beta$. To determine which, if any, of the PEGdi-DT hydrazide degradation products was responsible for the chemotaxis of EC migration, we individually tested the EC migratory influence of PEG, PEG-dihydrazide, and DT (Fig. 4c). Neither DT (5 mg/mL) nor PEG $(1 \mathrm{mg} / \mathrm{mL})$ treatment was found to significantly induce cell migration, but when cells were treated with PEG-dihydrazide at 1 $\mathrm{mg} / \mathrm{mL}$, migration was induced to roughly 2.4 times that of the medium control $(p<0.05)$.

We analyzed the effects of FGF- $\beta$, PEG-dihydrazide, and PEG on tubulogenesis by measuring tube length (Fig. $4 d)$. Figure $4 d$ demonstrates the length and structure of the tubules formed on the matrigel. We found that ECs treated with PEG-dihydrazide $(1 \mathrm{mg} / \mathrm{ml})$ organized into tubular-like structures; these results were compatible to those with FGF- $\beta$ treatment $(1 \mu \mathrm{g} / \mathrm{mL})$. In contrast, unorganized tubular structure was observed in ECs treated with PEG as well as with culture media. We used the interactive length-measuring feature of the microsuite to 


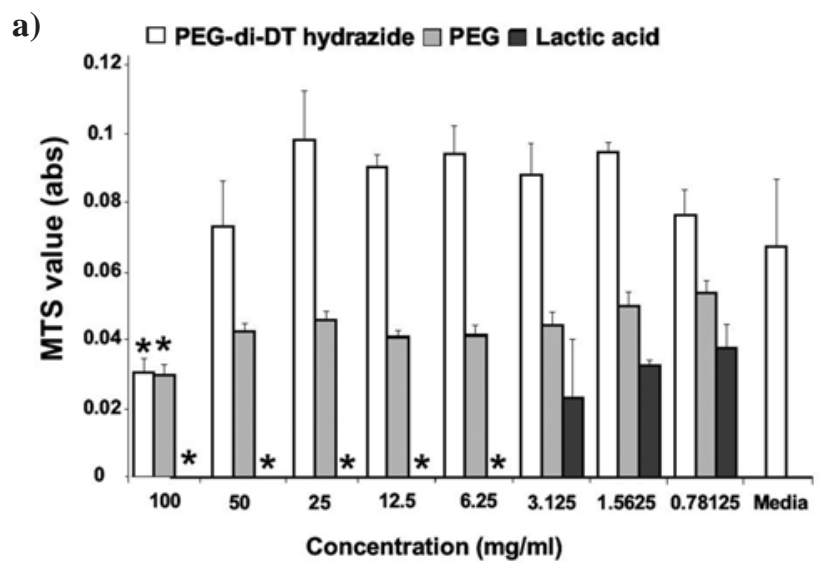

c)

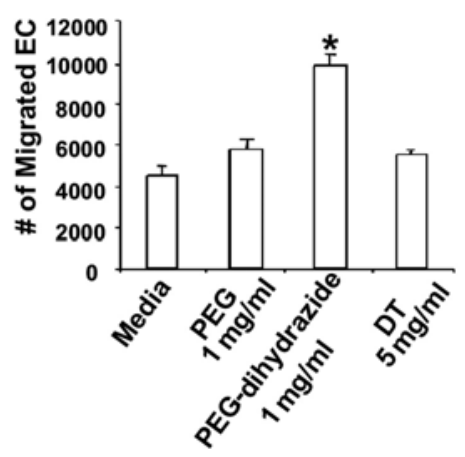

d)

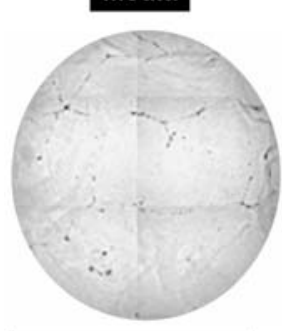

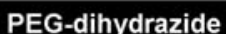

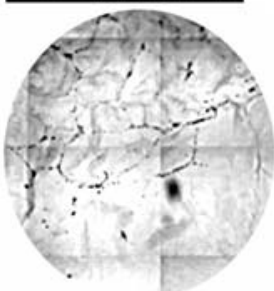

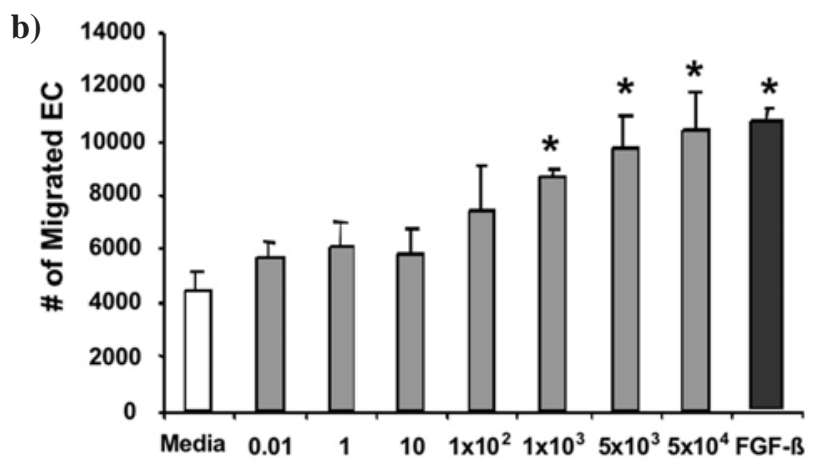

b)

Concentration of PEG -di-DT hydrazide $(\mu \mathrm{g} / \mathrm{ml})$
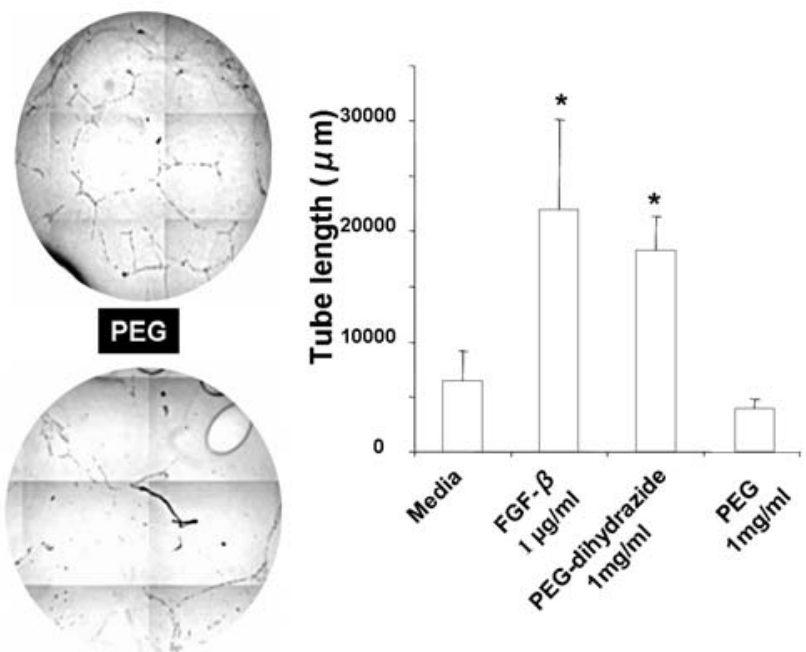

Figure 4. Effect of degradation products on cytotoxicity, EC migration, and tubulogenesis. a) Cytotoxicity of the degradation products analyzed with an MTS assay with RLF-6 cell line. Higher MTS values indicate greater cell viability. The effect of b) PEG-di-DT in a series of dilution and $\mathbf{c}$ ) its further degradation components, PEG, PEGdihydrazide, and DT on HAEC migration analyzed based on trans-well migration assay. d) Tubular structure and tube length measurements formatted by HAEC in Matrigel. FGF- $\beta$ and the media $(0 \mathrm{mg} / \mathrm{ml})$ were used as controls. $* P<0.05$ vs. the media.

determine the length of the formed tubules. Cells treated with FGF- $\beta(1 \mu \mathrm{g} / \mathrm{mL})$ or PEG-dihydrazide $(1 \mathrm{mg} / \mathrm{mL})$ had significantly longer tubes than those treated with the media $(p<0.05)$, indicating greater angiogenic activity. PEG $(1 \mathrm{mg} / \mathrm{mL})$ did not induce tubulogenesis.

Our H\&E histological observation of the subcutaneously implanted porous $10 \mathrm{D} / 8 \mathrm{P}$ hydrogel revealed a gradual increase in tissue infiltration over the 15-week period (Fig. 5a). One-week post implantation, we observed the presence of fibrous capsules. The empty white space indicated areas of non-degraded hydrogel that were most likely present because the polymers were dissolved during histological processing. Eight weeks after implantation, the scaffold was almost completely infiltrated. Our most interesting high-magnification observation was the presence of more blood vessels in the $10 \mathrm{D} / 8 \mathrm{P}$ scaffolds than in the poly (DTE carbonate) scaffolds (Fig. 5b). The vessels noted here were observed within the infiltrated tissue over the 15 weeks. "Fingers" of blood vessels appeared (indicated by the yellow arrows), which seemed to lead to tissue ingrowths towards the centre of the scaffold.

\section{Discussion}

In the present study, we fabricated a series of biodegradable hydrogels from poly(DTE-co- $x \%$ DT carbonate) crosslinked by $y \%$ poly(ethylene glycol) (PEG). The degradation profile of the resulting materials exemplified unique, controlled, predictable kinetics. The hydrogels and their degradation products exhibited suitable biocompatibility. Interestingly, PEG-di-DT hydrazide and its further degradation product PEG-dihydrazide stimulated in vitro angiogenic activities of endothelial cells. PEGcrosslinked poly(DTE-co-10\%DT carbonate) exhibited a strong potential to modulate angiogenesis.

The samples with high PEG content (at least 20\% PEG) exhibited accelerated mass loss, even with 25\% DT (Fig. $3 b)$. In previous studies, it has been shown that the incorporation of the hydrophilic PEG segments facilitates the solubilization of larger fragments and increases the rate of mass loss (Bezemer et al., 2000). The presence of PEG also increases the polymer's accessibility to water and, consequently, ensures degradation in the bulk of the matrices. 


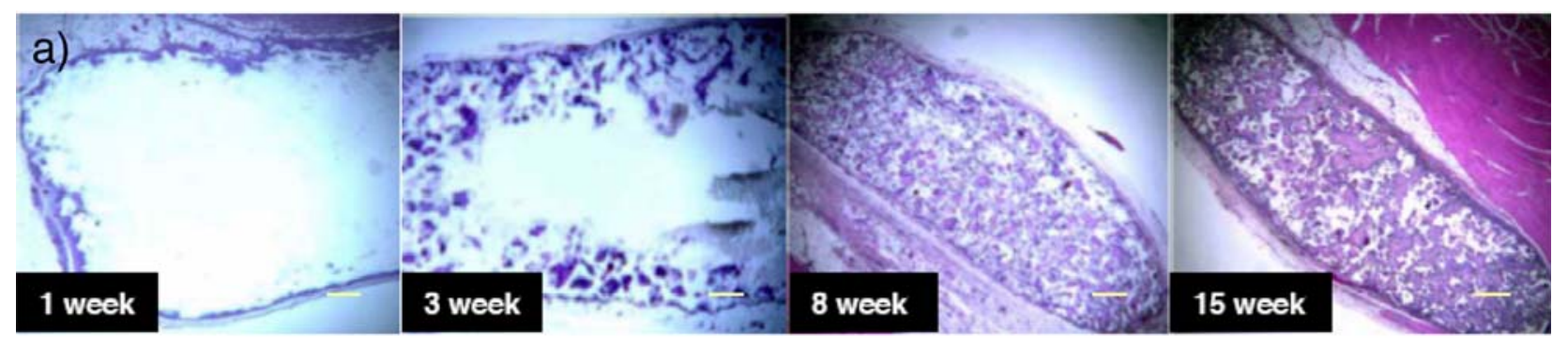

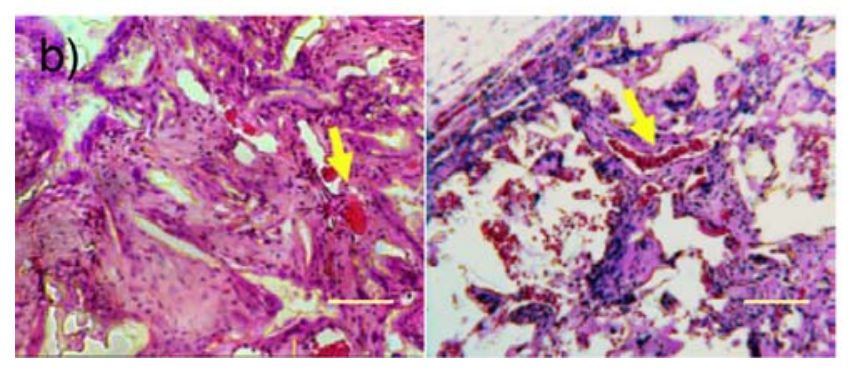

Figure 5. Tissue ingrowths and angiogenesis into the porous scaffolds implanted in vivo. H\&E histology showing a) tissue ingrowths into the scaffold (Bar $=100$ $\mu \mathrm{m})$ and b) vascularization of the poly DTE carbonate (left) and the porous 10D/8P (right) scaffolds with high magnification $(\mathrm{Bar}=100 \mu \mathrm{m})$. Yellow arrow indicates "fingers" of blood vessels leading tissue ingrowths to the scaffold. The images represent the flat surface from the mid-layer of the scaffold obtained after microtome slicing.
In contradiction to the finding of Zhao and Harris (Zhao and Harris, 1998), the PEG-crosslink density alone was not shown to significantly impact water uptake $(p>0.1)$ (Fig. 3a). We believe that this is due to the fact that increasing crosslinking is tied to increasing PEG content in the scaffolds, two factors that have opposite effects on water uptake (increased crosslinking, generally, decreases water uptake, whereas increasing PEG content increases water uptake).

The results from HPLC analysis of the incubation solution (Fig. 3c) indicate that the main mechanism of the initial degradation does not involve cleavage of the crosslinking unit but cleavage of the carbonate bonds in the polymer backbone, which are susceptible to water attack (Abramson, 2002). Since not all of the carboxylic acid groups in the polymer backbone are crosslinked, there are two sources for the DT release: the uncrosslinked carboxylic acids and PEG-di-DT hydrazide, which itself continues to degrade after being released as a product of degradation. The presence of the DT- $\mathrm{NHNH}_{2}$ peak signified that the crosslinking unit eventually degraded by cleavage of the amide bond between DT and PEGdihydrazide.

Only at the highest concentration $(100 \mathrm{mg} / \mathrm{mL})$ did PEG-di-DT hydrazide and PEG reduce RLF-6 viability when compared to the media $(p<0.05)$ (Fig. 4a). In their cytotoxicity study of the CHITOXANTM hydrogel, Chellat et al. noted that the inhibition of cell growth started at 5 $\mathrm{mg} / \mathrm{mL}$ when they treated its degradation product (Chellat et al., 2000). In other studies, PGA and PLA demonstrated cytotoxicity at concentrations of $87 \mathrm{mg} / \mathrm{mL}$ and $72 \mathrm{mg} /$ $\mathrm{mL}$, respectively (Taylor et al., 1994). We calculated that the theoretical maximum amount of PEG-di-DT hydrazide that could be released from the most quickly degrading hydrogel, $100 \mathrm{D} / 80 \mathrm{P}$, is roughly $36 \mathrm{mg}$. The minimum release from the slowest degrading hydrogel, 10D/8P, was calculated to be about 4-5 mg. Both of these calculations assume hydrogels are $8 \mathrm{~mm}$ in diameter and $2 \mathrm{~mm}$ thick. Within the range of all the above-mentioned concentrations, PEG-di-DT hydrazide did not demonstrate any cytotoxicity in comparison to the culture medium control.

It seems that the angiogenic effect of degradation products on in vitro EC migration and tube formation resides in the PEG-dihydrazide component. Previous studies on heparin affinity regulatory peptide (HARP) demonstrated that the $\mathrm{COOH}$ and $\mathrm{NH}_{2}$ terminals modulated angiogenesis in a dose-dependent and statistically significant manner (Papadimitriou et al., 2000; Papadimitriou et al., 2001). Researchers hypothesized that the two charged termini interact with cell surface molecules to facilitate HARP binding to its receptor and bring about a change in angiogenesis. Therefore, it is possible that the R-NHNH ${ }_{2}$ domains of the degradation products PEG-diDT hydrazide and PEG-dihydrazide are responsible for modulating EC migration and tubulogenesis. Further studies are necessary to confirm this hypothesis.

The in vitro cytotoxicity was assayed to investigate the biocompatibility of the degradation products. This in vitro assay provided a rapid and easy screening of polymer/cell interactions without the interference from wound response normally seen in vivo (Attawia et al., 1995). The MTS assay measured cell viability to indicate not only the potency of the compound, but also the minimum dose responsible for inducing cell death. However, in vitro assays cannot confirm the compound's complete biocompatibility because they lack many of the complex physiological parameters provided by an in vivo environment. Nevertheless, our in vivo histological evaluation also indicated no noticeable cytotoxicity for the hydrogels or their degradation products.

Angiogenesis in matrixes of biomaterial scaffolds is a complex multi-step process that includes the initial steps of matrix degradation around endothelial cells, migration of endothelial cells into newly formed interstices, and proliferation of migrating endothelial cells (mainly at growth sprouts). The final step in angiogenesis includes the interaction between adjacent endothelial cells to form 
a new lumen (i.e. tubulogenesis) and supporting basement membrane (extracellular matrix) and the simultaneous reinforcement of endothelial cells provided by muscle cell pericytes (Gupta and Qin, 2003). It has been established that controlling the degree of angiogenesis and the amount of biomaterial implant vascularization leads to better implant integration from autologous tissue infiltration of the graft. If a polymer scaffold has angiogenic properties, then this increases the likelihood that the implant will be successful since vessel arborization is integral to wound healing and graft survival. Researchers have been engineering new ways to increase angiogenesis by using a combination of biomaterials with controlled drug release systems, co-cultures with ECM and growth factor producing cells, and growth factor injections. The new class of biomaterials examined in this study, however, demonstrates a strong potential to modulate angiogenesis without being combined with the aforementioned additional systems.

One area in which this angiogenic hydrogel scaffold is applicable is in the musculoskeletal system, where vascularization is a key factor for the development of normal bone and cartilage (Ballara et al., 1999). The formation of new blood vessels is crucial during bone development in the embryo, rapid bone growth, and fracture healing, all of which involve vascularization of cartilage (Gerber and Ferrara, 2000). During endochondral bone ossification (the formation of bone from cartilage), calcified cartilage allows for vascular invasion, which initiates the replacement of cartilage by bone (Harper and Klagsbrun, 1999). The cartilage, usually an avascular tissue, hypertrophies and becomes infiltrated with blood vessels which erode the tissue, producing a scaffold on which osteoblasts settle and produce bone (Ballara et al., 1999). During fracture repair, angiogenesis is essential for proper healing (Hausman et al., 2001) and occurs in response to bone injury or pathological conditions (Gerber and Ferrara, 2000). In particular, the anigiogenic property of these hydrogels would be useful to regenerate bone tissue for defects larger than $2 \mathrm{~mm}$.

The effect of the DT content and PEG-crosslink density on in vivo angiogenic activities is a consideration for future examination. Therefore, we plan to expand the hydrogel types by varying the DT content and/or PEG-crosslink density for the next set of in vivo studies. Additional in vivo models, including the rabbit cornea assay and the chick chorioallantoic membrane assay, will be used for these future studies.

\section{Conclusion}

A family of PEG-crosslinked, tyrosine-derived hydrogels was synthesized based on poly(DTE-co- $x \%$ DT carbonate), which provided sites for chemical crosslinking with PEGdihydrazide. We generated a series of hydrogels with varying DT contents and crosslinking levels. The porous 10D/8P hydrogel scaffolds possessed fairly interconnected macro- and micro-pores. DT and PEG contents were the major chemistry factors contributing to water uptake and degradation. The hydrogels degraded into the three major release products: DT, DTE, and PEG-di-DT hydrazide. PEG-di-DT hydrazide was found to further degrade and release DT, which logically leads to the possibility that hydrazine may also be released. PEG-di-DT hydrazide was shown to induce in vitro EC migration and tube formation while exhibiting no noticeable cytotoxicity except at the highest concentration $(100 \mathrm{mg} / \mathrm{ml})$. Interestingly, PEGdihydrazide was equally as active as PEG-di-DT hydrazide at the same concentrations. Therefore, it is likely that the biological activity of PEG-di-DT hydrazide resides in the PEG-dihydrazide component. In our in vivo $\mathrm{H} \& \mathrm{E}$ histological evaluation, the 10D/8P hydrogel demonstrated a gradual increase in the tissue infiltration over the 15week time period and seemed to effectively promote blood vessel growth. These results indicate that PEG-crosslinked poly(DTE-co-10\%DT carbonate) shows great promise as a biomaterial that could ensure proper vascularization around the implantation site and aid in preserving implant patency by improving angiogenesis.

\section{Acknowledgements}

We thank Dr. J.R. Parsons for histological analysis and Mrs. Esther Adler for experimental assistance. This work was supported by NIH Grant EB 003057 and the New Jersey Center for Biomaterials.

\section{References}

Abramson S (2002) Selected bulk and surface properties and biocompatibility of a new class of tyrosinederived polycarbonates. R.A161 2002, Biomedical Engineering, Rutgers-The State University of New Jersey, New Jersey Center for Biomaterials, 145 Bevier Road, Piscataway, New Jersey 08854.

Attawia MA, Uhrich KE, Botchwey E, Fan M, Langer R, Laurencin CT (1995) Cytotoxicity testing of poly(anhydride-co-imides) for orthopedic applications. J Biomed Mater Res 29:1233-1240.

Bachetti T, Morbidelli L (2000) Endothelial cells in culture: a model for studying vascular functions. Pharmacol Res 42: 9-19.

Ballara SC, Miotla JM, Paleolog EM (1999) New vessels, new approaches: angiogenesis as a therapeutic target in musculoskeletal disorders. Int J Exp Pathol 80: 235-250.

Bernardini D, Nasulewic A, Mazur A, Maier JA (2005) Magnesium and microvascular endothelial cells: a role in inflammation and angiogenesis. Front Biosci 10: 11771182.

Bezemer JM, Oude Weme P, Grijpma DW, Dijkstra PJ, van Blitterswijk CA, Feijen J (2000) Amphiphilic poly(ether ester amide) multiblock copolymers as biodegradable matrices for the controlled release of proteins. J Biomed Mater Res 52: 8-17.

Bishop ET, Bell GT, Bloor S, Broom IJ, Hendry NF, Wheatley DN (1999) An in vitro model of angiogenesis: basic features. Angiogenesis 3: 335-344. 
Black AF, Hudon V, Damour O, Germain L, Auger FA (1999) A novel approach for studying angiogenesis: a human skin equivalent with a capillary-like network. Cell Biol Toxicol 15: 81-90.

Cassell OC, Hofer SO, Morrison WA, Knight KR (2002) Vascularisation of tissue-engineered grafts: the regulation of angiogenesis in reconstructive surgery and in disease states. Br J Plast Surg 55: 603-610.

Chellat F, Tabrizian M, Dumitriu S, Chornet E, Magny $\mathrm{P}$, Rivard $\mathrm{CH}$, Yahia L (2000) In vitro and in vivo biocompatibility of chitosan-xanthan polyionic complex. J Biomed Mater Res 51: 107-116.

Eckardt H, Bundgaard KG, Christensen KS, Lind M, Hansen ES, Hvid I (2003) Effects of locally applied vascular endothelial growth factor (VEGF) and VEGFinhibitor to the rabbit tibia during distraction osteogenesis. J Orthop Res 21: 335-340.

Ertel SI, Kohn J (1994) Evaluation of a series of tyrosine-derived polycarbonates as degradable biomaterials. J Biomed Mater Res 28: 919-930.

Folkman J, Shing Y (1992) Angiogenesis. J Biol Chem 267: 10931-10934.

Gerber HP, Ferrara N (2000) Angiogenesis and bone growth. Trends Cardiovasc Med 10: 223-228.

Gupta MK, Qin RY (2003) Mechanism and its regulation of tumor-induced angiogenesis. World $\mathrm{J}$ Gastroenterol 9: 1144-1155.

Harper J, Klagsbrun M (1999) Cartilage to boneangiogenesis leads the way. Nat Med 5: 617-618.

Hausman MR, Schaffler MB, Majeska RJ (2001) Prevention of fracture healing in rats by an inhibitor of angiogenesis. Bone 29: 560-564.

Jain RK, Schlenger K, Hockel M, Yuan F (1997) Quantitative angiogenesis assays: progress and problems. Nat Med 3: 1203-1208.

James K, Levene H, Parsons JR, Kohn J (1999) Small changes in polymer chemistry have a large effect on the bone-implant interface: evaluation of a series of degradable tyrosine-derived polycarbonates in bone defects. Biomaterials 20: 2203-2212.

Kidd KR, Nagle RB, Williams SK (2002) Angiogenesis and neovascularization associated with extracellular matrix-modified porous implants. J Biomed Mater Res 59: 366-377.

Kim SH, Won CY, Chu CC (1999) Synthesis and characterization of dextran-maleic acid based hydrogel. J Biomed Mater Res 46: 160-170.

Labazzo K (2004) Hydrogels Fabricated from PEGCrosslinked Tyrosine-Derived Polycarbonates and their Angiogenic Potential for Tissue Engineering. Rutgers-The State University of New Jersey, Biomedical Engineering.

Levene H (1999) Analysis of tyrosine-derived novel synthetic polymer scaffold devices for guided tissue regeneration. R.L657 1999, Biomedical Engineering, Rutgers-The State University of New Jersey, New Jersey Center for Biomaterials, 145 Bevier Road, Piscataway, New Jersey 08854.

Lutolf MP, Hubbell JA (2005) Synthetic biomaterials as instructive extracellular microenvironments for morphogenesis in tissue engineering. Nat Biotechnol 23: 47-55.
Malich G, Markovic B, Winder C (1997) The sensitivity and specificity of the MTS tetrazolium assay for detecting the in vitro cytotoxicity of 20 chemicals using human cell lines. Toxicology 124: 179-192.

Montanez E, Casaroli-Marano RP, Vilaro S, Pagan R (2002) Comparative study of tube assembly in threedimensional collagen matrix and on Matrigel coats. Angiogenesis 5: 167-172.

Papadimitriou E, Heroult M, Courty J, Polykratis A, Stergiou C, Katsoris P (2000) Endothelial cell proliferation induced by HARP: implication of $\mathrm{N}$ or $\mathrm{C}$ terminal peptides. Biochem Biophys Res Commun 274: 242-248.

Papadimitriou E, Polykratis A, Courty J, Koolwijk P, Heroult M, Katsoris P (2001) HARP induces angiogenesis in vivo and in vitro: implication of $\mathrm{N}$ or $\mathrm{C}$ terminal peptides. Biochem Biophys Res Commun 282: 306-313.

Sieminski AL, Gooch KJ (2000) Biomaterialmicrovasculature interactions. Biomaterials 21: 22322241.

Sung HJ, Meredith C, Johnson C, Galis ZS (2004) The effect of scaffold degradation rate on three-dimensional cell growth and angiogenesis. Biomaterials 25: 5735-5742.

Tabata Y, Nagano A, Muniruzzaman M, Ikada Y (1998) In vitro sorption and desorption of basic fibroblast growth factor from biodegradable hydrogels. Biomaterials 19: 1781-1789.

Taylor MS, Daniels AU, Andriano KP, Heller J (1994) Six bioabsorbable polymers: in vitro acute toxicity of accumulated degradation products. J Appl Biomater 5: 151-157.

Tello-Montoliu A, Patel JV, Lip GY (2006) Angiogenin: a review of the pathophysiology and potential clinical applications. J Thromb Haemost 4: 1864-1874.

Yla-Herttuala S, Rissanen TT, Vajanto I, Hartikainen J (2007) Vascular endothelial growth factors: biology and current status of clinical applications in cardiovascular medicine. J Am Coll Cardiol 49: 1015-1026.

Yu C, Kohn J (1999) Tyrosine-PEG-derived poly(ether carbonate)s as new biomaterials. Part I: synthesis and evaluation. Biomaterials 20: 253-264.

Zhao X, Harris JM (1998) Novel degradable poly(ethylene glycol) hydrogels for controlled release of protein. J Pharm Sci 87: 1450-1458.

\section{Discussion with Reviewers}

S. Fuchs: Individual cell types react differently to cytotoxic compounds. The authors tested the cytotoxic reaction of fibroblasts towards one of the degradation products PEGdi-DT hydrazide. How do endothelial cells react towards the tested compound or in extraction test according to the ISO guideline to the mixture of the single degradation products of the proposed hydrogels?

Authors: We agree with the reviewer's comment that ISObased cytotoxic values of individual cell types in response to PEG-di-DT might be different. Rat lung fibroblasts (RLF-6) were used for our cytotoxicity test because this fibroblast type has been used extensively in previous studies investigating the cytotoxic effects of biomaterial compounds. As we described in the Results section, since 
DT and DTE demonstrated no noticeable cytotoxicity or angiogenic effect in our previous studies (Ertel and Kohn, 1994; James et al., 1999; Yu and Kohn, 1999), the present study focused on the cytotoxic and angiogenic effect of PEG-di-DT hydrazide and its further degradation product, PEG-dihydrazide. The results from the assays of EC migration under a series of conditions varying concentration and type of model degradation compounds, including PEG-di-DT hydrazide, demonstrated indirectly that all the tested compounds had no cytotoxic effect upon human aortic endothelial cells within the tested range of concentration. The range of concentration of an individual compound was determined following the guidelines established in ISO 10993 and the US FDA blue book memorandum (\#G95-1). Multiple lines of evidence from the tests of in vitro tubulogenesis and in vivo tissue ingrowth/angiogeneisis point out the fact that all of the single and mixture compounds are not cytotoxic.

S. Fuchs: In vivo evaluation: What was the reason to choose specifically $10 \mathrm{D} / 8 \mathrm{P}$ hydrogels for in vivo evaluation? Do the authors have any additional information about the influence of the desaminotyrosyl tyrosine content or the cross-link density on the outcome of the vascularization process in vivo or in vitro? This information would be essential to understand how PEGcross-linked tyrosine-derived polycarbonates hydrogels biomaterial per se could modulate angiogenesis in further applications.

Authors: In this study, we used only $10 \mathrm{D} / 8 \mathrm{P}$ and poly(DTE carbonate) scaffolds for implantation. However, we plan to perform further in vivo studies on the implantation of other types of hydrogels with varying desaminotyrosyl tyrosine contents and/or cross link densities, as the reviewer suggested, to better understand how they influence angiogenic processes. We agree with the reviewer's comment that this information would be valuable in improving our understanding of how PEGcross-linked tyrosine-derived polycarbonate hydrogel biomaterials could modulate angiogenesis in further applications.

D. Eglin: What is the application that the authors foreseen for such polymeric scaffold? Would it be in bone tissue engineering?

Authors: One area in which this angiogenic hydrogel scaffold is applicable is in the musculoskeletal system, where vascularization is a key factor for the development of normal bone and cartilage (Ballara et al., 1999). The formation of new blood vessels is crucial during bone development in the embryo, rapid bone growth, and fracture healing, all of which involve vascularization of cartilage (Gerber and Ferrara, 2000). During endochondral bone ossification (formation of bone from cartilage), calcified cartilage allows for vascular invasion, which initiates the replacement of cartilage by bone (Harper and Klagsbrun, 1999). The cartilage, usually an avascular tissue, hypertrophies and becomes infiltrated with blood vessels which erode the tissue, producing a scaffold on which osteoblasts settle and produce bone (Ballara et al., 1999). During fracture repair, angiogenesis is essential for proper healing (Hausman et al., 2001) and occurs in response to bone injury or pathological conditions (Gerber and Ferrara, 2000). In particular, the anigiogenic property of these hydrogels would be useful to regenerate bone tissue for defects larger than $2 \mathrm{~mm}$. 